\title{
Accurate and Efficient Prediction of NMR Parameters of \\ Condensed-Phase Systems with the Generalized Energy-Based Fragmentation Method
}

Dongbo Zhao ${ }^{\dagger, \ddagger}$, Xiaoling Shen ${ }^{\dagger}$, Zheng Chen ${ }^{\dagger}$, Wei Li ${ }^{\dagger}$, Hao Dong ${ }^{\dagger, l, *}$, Shuhua $\mathrm{Li}^{\dagger}{ }^{\dagger, *}$

†School of Chemistry and Chemical Engineering, Nanjing University, 210023, People’s Republic of China

¥Kuang Yaming Honors School, Nanjing University, 210023, People’s Republic of China "Institute for Brain Sciences, Nanjing University, 210023, People's Republic of China

*Corresponding authors. Emails: shuhua@nju.edu.cn and donghao@nju.edu.cn 
The Principle of Inclusion and Exclusion. For brevity, we will use a simple model system (ABC) to illustrate the process in GEBF as shown in Figure S1. A, B, and C each contains a few atoms or groups. We assume that the target system $\mathrm{ABC}$ (with three fragments $\mathrm{A}, \mathrm{B}$, and $\mathrm{C}$ ) can be divided into two (primitive) subsystems $\mathrm{AB}$ and $\mathrm{BC}$ in step 1, according to a distance threshold. The coefficients for the two primitive subsystems are +1 . Then an extra (derivative) subsystem $\mathrm{B}$ is generated (due to the overlapping of $\mathrm{AB}$ and $\mathrm{BC}$ ) in step 2. The coefficient for subsystem $\mathrm{B}$ is determined to be -1 according to the principle of inclusion and exclusion. Finally, the total energy of the target system $\mathrm{ABC}$ is approximated as: $E_{\mathrm{ABC}} \approx E_{\mathrm{AB}}+E_{\mathrm{BC}}-E_{\mathrm{B}}$.

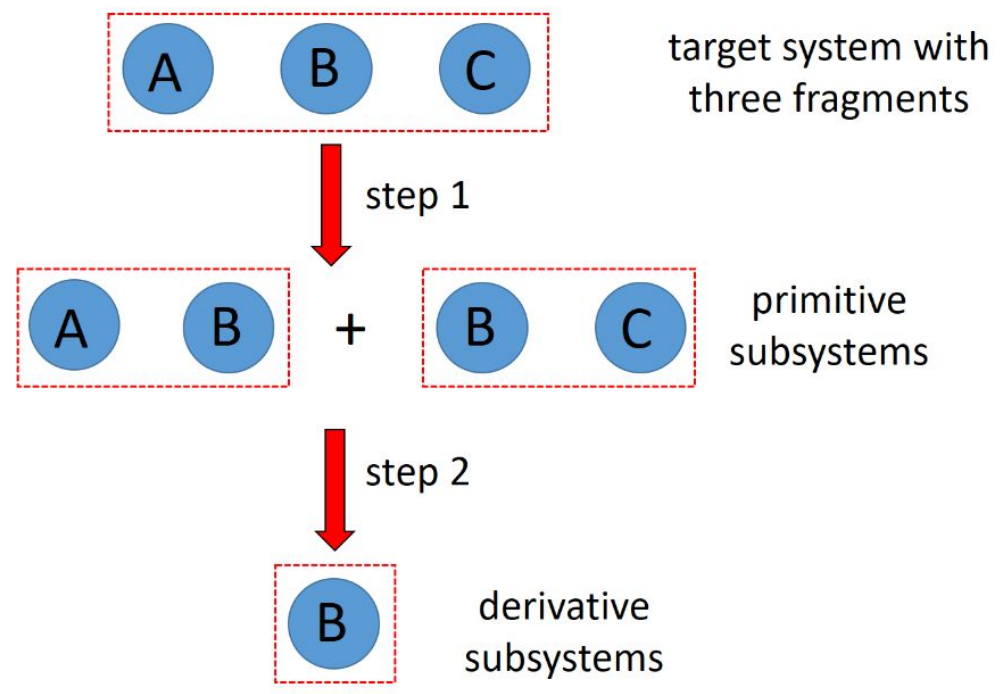

Figure S1. The fragmentation process of GEBF for molecular systems as an illustration. 


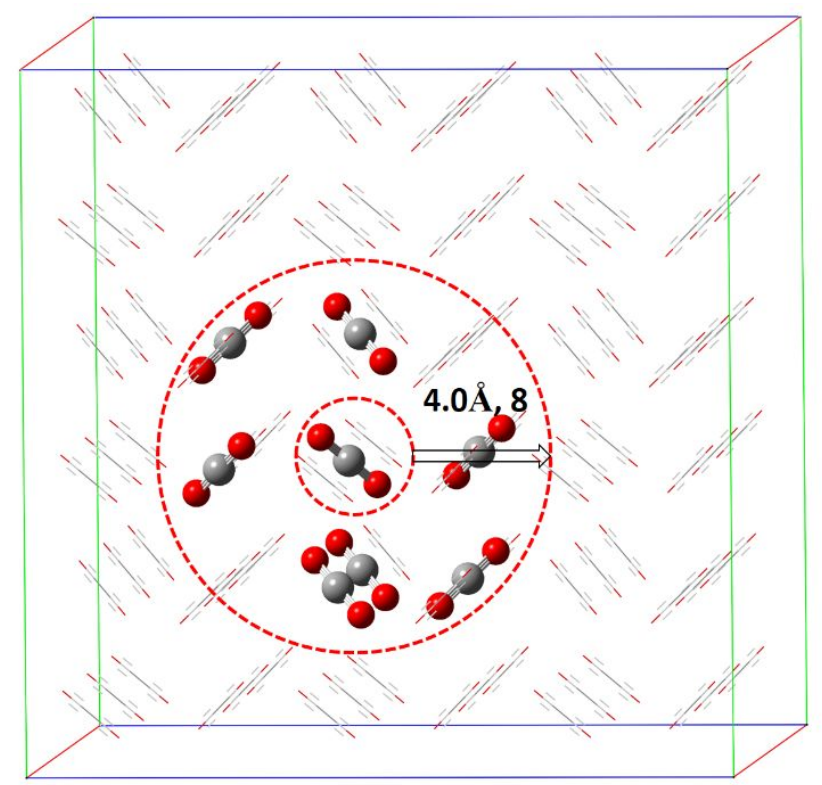

Figure S2. One of the subsystems of carbon dioxide crystals generated with the "molecule-based" fragmentation strategy in PBC-GEBF. Eight $\mathrm{CO}_{2}$ molecules (in ball mode) in a subsystem are embedded in background point charges (in stick mode) of atoms beyond the subsystem in a supercell $K$. The coefficient of this subsystem is +1 . The compensation field charges are added on the boundary surfaces and they are not shown. Color code: $\mathrm{C}$ in grey and $\mathrm{O}$ in red. 
Table S1. Comparison of optimized and experimental lattice parameters of crystals with small molecules. CCDC reference codes are also listed.

\begin{tabular}{|c|c|c|c|c|c|c|c|}
\hline uracil & monoclinic & \multicolumn{2}{|c|}{ CCDC: 1278441} & \multirow{2}{*}{$\begin{array}{l}\text { guanine } \\
\text { Lattice }\end{array}$} & \multirow{2}{*}{$\begin{array}{c}\text { monoclinic } \\
\text { exp. }\end{array}$} & \multicolumn{2}{|c|}{ CCDC:618636 } \\
\hline Lattice & exp. & relaxed & $\Delta(\%)$ & & & relaxed & $\Delta(\%)$ \\
\hline$a(\AA)$ & 11.938 & 11.897 & -0.3 & $a(\AA)$ & 3.553 & 3.607 & 1.5 \\
\hline$b(\AA)$ & 12.376 & 12.444 & 0.5 & $b(\AA)$ & 9.693 & 9.719 & 0.3 \\
\hline$c(\AA)$ & 3.655 & 3.590 & -1.8 & $c(\AA)$ & 16.345 & 16.417 & 0.4 \\
\hline$\alpha\left(^{\circ}\right)$ & 90.0 & 89.8 & -0.2 & $\alpha\left(^{\circ}\right)$ & 90.0 & 89.9 & -0.1 \\
\hline$\beta\left(^{\circ}\right)$ & 120.9 & 119.5 & -1.1 & $\beta\left(^{\circ}\right)$ & 95.7 & 95.8 & 0.0 \\
\hline$\gamma\left({ }^{\circ}\right)$ & 90.0 & 90.2 & 0.2 & $\gamma\left({ }^{\circ}\right)$ & 90.0 & 90.4 & 0.4 \\
\hline Vol. $\left(\AA^{3}\right)$ & 463.4 & 462.5 & -0.2 & Vol. $\left(\AA^{3}\right)$ & 560.1 & 572.5 & 2.2 \\
\hline cytosine & orthorhombic & \multicolumn{2}{|c|}{ CCDC: 1134840} & thymine & monoclinic & \multicolumn{2}{|c|}{ CCDC: 136916} \\
\hline Lattice & exp. & relaxed & $\Delta(\%)$ & Lattice & exp. & relaxed & $\Delta(\%)$ \\
\hline$a(\AA)$ & 11.938 & 11.897 & -0.3 & $a(\AA)$ & 12.889 & 12.724 & -1.3 \\
\hline$b(\AA)$ & 12.376 & 12.444 & 0.5 & $b(\AA)$ & 6.852 & 6.848 & -0.1 \\
\hline$c(\AA)$ & 3.655 & 3.590 & -1.8 & $c(\AA)$ & 6.784 & 6.713 & -1.1 \\
\hline$\alpha\left(^{\circ}\right)$ & 90.0 & 89.8 & -0.2 & $\alpha\left(^{\circ}\right)$ & 90.0 & 90.2 & 0.3 \\
\hline$\beta\left(^{\circ}\right)$ & 120.9 & 119.5 & -1.1 & $\beta\left(^{\circ}\right)$ & 104.9 & 105.2 & 0.3 \\
\hline$\gamma\left({ }^{\circ}\right)$ & 90.0 & 90.2 & 0.2 & $\gamma\left({ }^{\circ}\right)$ & 90.0 & 90.1 & 0.2 \\
\hline Vol. $\left(\AA^{3}\right)$ & 463.4 & 462.5 & -0.2 & Vol. $\left(\AA^{3}\right)$ & 578.9 & 564.5 & -2.5 \\
\hline adenine & monoclinic & \multicolumn{2}{|c|}{ CCDC: 652573} & borane & orthorhombic & \multicolumn{2}{|c|}{ CCDC:132999 } \\
\hline Lattice & exp. & relaxed & $\Delta(\%)$ & Lattice & exp. & relaxed & $\Delta(\%)$ \\
\hline$a(\AA)$ & 7.891 & 7.904 & 0.2 & $a(\AA)$ & 5.395 & 5.366 & -0.5 \\
\hline$b(\AA)$ & 22.242 & 22.241 & 0.0 & $b(\AA)$ & 4.887 & 5.152 & 5.4 \\
\hline$c(\AA)$ & 7.448 & 7.434 & -0.2 & $c(\AA)$ & 4.986 & 4.955 & -0.6 \\
\hline$\alpha\left(^{\circ}\right)$ & 90.0 & 89.5 & -0.5 & $\alpha\left(^{\circ}\right)$ & 90.0 & 90.0 & 0.0 \\
\hline$\beta\left(^{\circ}\right)$ & 113.193 & 113.0 & -0.2 & $\beta\left(^{\circ}\right)$ & 90.0 & 90.0 & 0.0 \\
\hline$\gamma\left({ }^{\circ}\right)$ & 90.0 & 90.1 & 0.1 & $\gamma\left({ }^{\circ}\right)$ & 90.0 & 90.0 & 0.0 \\
\hline Vol. $\left(\AA^{3}\right)$ & 1201.6 & 1202.9 & 0.1 & Vol. $\left(\AA^{3}\right)$ & 131.5 & 137.0 & 4.2 \\
\hline L-glycine & monoclinic & \multicolumn{2}{|c|}{ CCDC: 1169354} & L-valine & monoclinic & \multicolumn{2}{|c|}{ CCDC: 1208817} \\
\hline Lattice & exp. & relaxed & $\Delta(\%)$ & Lattice & exp. & relaxed & $\Delta(\%)$ \\
\hline$a(\AA)$ & 5.102 & 5.155 & 1.0 & $a(\AA)$ & 9.71 & 9.69 & -0.3 \\
\hline$b(\AA)$ & 11.971 & 11.944 & -0.2 & $b(\AA)$ & 5.27 & 5.27 & 0.1 \\
\hline$c(\AA)$ & 5.457 & 5.496 & 0.7 & $c(\AA)$ & 12.06 & 11.86 & -1.6 \\
\hline$\alpha\left(^{\circ}\right)$ & 90.0 & 90.0 & 0.0 & $\alpha\left(^{\circ}\right)$ & 90.0 & 90.1 & 0.1 \\
\hline$\beta\left(^{\circ}\right)$ & 111.7 & 111.3 & -0.4 & $\beta\left(^{\circ}\right)$ & 90.8 & 90.8 & 0.0 \\
\hline
\end{tabular}




\begin{tabular}{|c|c|c|c|c|c|c|c|c|}
\hline$\gamma\left({ }^{\circ}\right)$ & 90.0 & 89.5 & \multicolumn{2}{|c|}{-0.5} & $\gamma\left(\left(^{\circ}\right)\right.$ & 90.0 & 90.1 & 0.1 \\
\hline Vol. $\left(\AA^{3}\right)$ & 309.7 & 315.3 & \multicolumn{2}{|c|}{1.8} & Vol. $\left(\AA^{3}\right)$ & 617.1 & 606.0 & -1.8 \\
\hline L-serine & rthorhombic & \multicolumn{2}{|c|}{ CCDC:263153 } & \multicolumn{2}{|r|}{ L-alanine } & orthorhombic & \multicolumn{2}{|c|}{ CCDC: 1203203} \\
\hline Lattice & exp. & relaxed & $\Delta(\%$ & & Lattice & exp. & relaxed & $\Delta(\%)$ \\
\hline$a(\AA)$ & 8.599 & 8.661 & \multicolumn{2}{|c|}{0.7} & $a(\AA)$ & 6.025 & 5.941 & -1.4 \\
\hline$b(\AA)$ & 9.348 & 9.332 & \multicolumn{2}{|c|}{-0.2} & $b(\AA)$ & 12.324 & 12.262 & -0.5 \\
\hline$c(\AA)$ & 5.618 & 5.626 & \multicolumn{2}{|c|}{0.2} & $c(\AA)$ & 5.783 & 5.810 & 0.5 \\
\hline$\alpha\left(^{\circ}\right)$ & 90.0 & 89.8 & \multicolumn{2}{|c|}{-0.2} & $\alpha\left(^{\circ}\right)$ & 90.0 & 89.9 & -0.1 \\
\hline$\beta\left(^{\circ}\right)$ & 90.0 & 90.0 & \multicolumn{2}{|c|}{0.0} & $\beta\left(^{\circ}\right)$ & 90.0 & 90.0 & 0.0 \\
\hline$\gamma\left({ }^{\circ}\right)$ & 90.0 & 90.0 & \multicolumn{2}{|c|}{0.0} & $\gamma\left({ }^{\circ}\right)$ & 90.0 & 89.9 & -0.1 \\
\hline Vol. $\left(\AA^{3}\right)$ & 451.6 & 454.8 & \multicolumn{2}{|c|}{0.7} & Vol. $\left(\AA^{3}\right)$ & 429.4 & 423.3 & -1.4 \\
\hline aspirin & monoclinic & \multicolumn{2}{|c|}{ CCDC:610952 } & & imidazole & nonoclinic & \multicolumn{2}{|c|}{ CCDC:1180146 } \\
\hline Lattice & exp. & relaxed & $\Delta(\%)$ & & Lattice & exp. & relaxed & $\Delta(\%)$ \\
\hline$a(\AA)$ & 11.278 & 11.124 & -1.4 & & $a(\AA)$ & 7.569 & 7.571 & 0.0 \\
\hline$b(\AA)$ & 6.552 & 6.509 & -0.7 & & $b(\AA)$ & 5.366 & 5.365 & 0.0 \\
\hline$c(\AA)$ & 11.274 & 11.268 & -0.1 & & $c(\AA)$ & 9.785 & 9.736 & -0.5 \\
\hline$\alpha\left(^{\circ}\right)$ & 90.0 & 90.1 & 0.1 & & $\alpha\left(^{\circ}\right)$ & 90.0 & 90.1 & 0.1 \\
\hline$\beta\left(^{\circ}\right)$ & 95.8 & 96.3 & 0.6 & & $\beta\left(^{\circ}\right)$ & 119.1 & 118.4 & -0.6 \\
\hline$\gamma\left({ }^{\circ}\right)$ & 90.0 & 90.1 & 0.1 & & $\gamma\left({ }^{\circ}\right)$ & 90.0 & 89.9 & -0.1 \\
\hline Vol. $\left(\AA^{3}\right)$ & 828.7 & 810.8 & -2.2 & & Vol. $\left(\AA^{3}\right)$ & 347.3 & 347.8 & 0.2 \\
\hline urea & tetragonal & CCDC: 1 & 278498 & & carbon dioxide & cubic & CCDC: $\mathrm{u}$ & lavailable \\
\hline Lattice & exp. & relaxed & $\Delta(\%$ & & Lattice & exp. & relaxed & $\Delta(\%)$ \\
\hline$a(\AA)$ & 5.565 & 5.572 & & & $a(\AA)$ & 5.63 & 5.68 & \\
\hline$b(\AA)$ & 5.565 & 5.572 & & & $b(\AA)$ & 5.63 & 5.68 & \\
\hline$c(\AA)$ & 4.684 & 4.692 & & & $c(\AA)$ & 5.63 & 5.68 & \\
\hline$\alpha\left(^{\circ}\right)$ & 90.0 & 90.0 & & & $\alpha\left(^{\circ}\right)$ & 90.0 & 89.8 & \\
\hline$\beta\left(^{\circ}\right)$ & 90.0 & 90.0 & & & $\beta\left(^{\circ}\right)$ & 90.0 & 89.8 & \\
\hline$\gamma\left({ }^{\circ}\right)$ & 90.0 & 90.0 & & & $\gamma\left({ }^{\circ}\right)$ & 90.0 & 89.8 & \\
\hline Vol. $\left(\AA^{3}\right)$ & 145.1 & 145.7 & & & Vol. $\left(\AA^{3}\right)$ & 178.5 & 183.3 & \\
\hline 3-deazaauraci & orthorh & mbic & CDC: 11 & 1055 & acetylene & cubic & CCDC: 1 & 00662 \\
\hline Lattice & $\mathrm{ex}]$ & & laxed & $\Delta(\%)$ & Lattice & exp. & relaxed & $\Delta(\%)$ \\
\hline$a(\AA)$ & & 8.638 & 8.667 & 0.3 & $a(\AA)$ & 6.094 & 6.120 & 0.4 \\
\hline$b(\AA)$ & & 5.315 & 5.331 & 0.3 & $b(\AA)$ & 6.094 & 6.120 & 0.4 \\
\hline$c(\AA)$ & & 1.221 & 11.236 & 0.1 & $c(\AA)$ & 6.094 & 6.120 & 0.4 \\
\hline$\alpha\left(^{\circ}\right)$ & & 90.0 & 90.0 & 0.0 & $\alpha\left(^{\circ}\right)$ & 90.0 & 89.9 & -0.1 \\
\hline$\beta\left(^{\circ}\right)$ & & 90.0 & 90.1 & 0.1 & $\beta\left(^{\circ}\right)$ & 90.0 & 89.9 & -0.1 \\
\hline$\gamma\left({ }^{\circ}\right)$ & & 90.0 & 90.1 & 0.1 & $\gamma\left({ }^{\circ}\right)$ & 90.0 & 89.9 & -0.1 \\
\hline Vol. $\left(\AA^{3}\right)$ & & 515.2 & 519.1 & 0.8 & Vol. $\left(\AA^{3}\right)$ & 226.3 & 229.2 & 1.3 \\
\hline
\end{tabular}




\begin{tabular}{cccccccc} 
ethylene & monoclinic & \multicolumn{2}{c}{ CCDC:1150502 } & L-glutamic acid & orthorhombic & \multicolumn{2}{c}{ CCDC:1206530 } \\
Lattice & exp. & relaxed & $\Delta(\%)$ & Lattice & exp. & relaxed & $\Delta(\%)$ \\
$a(\AA)$ & 4.626 & 4.639 & 2.8 & $a(\AA)$ & 10.282 & 10.285 & 0.0 \\
$b(\AA)$ & 6.620 & 6.629 & 1.3 & $b(\AA)$ & 8.779 & 8.788 & 0.1 \\
$c(\AA)$ & 4.067 & 4.073 & 1.5 & $c(\AA)$ & 7.068 & 7.071 & 0.0 \\
$\alpha\left(^{\circ}\right)$ & 90.0 & 89.9 & -0.1 & $\alpha\left(^{\circ}\right)$ & 90.0 & 90.0 & 0.0 \\
$\beta\left(^{\circ}\right)$ & 94.4 & 94.3 & -0.1 & $\beta\left(^{\circ}\right)$ & 90.0 & 90.0 & 0.0 \\
$\gamma\left({ }^{\circ}\right)$ & 90.0 & 90.3 & 0.3 & $\gamma\left(^{\circ}\right)$ & 90.0 & 90.0 & 0.0 \\
Vol. $\left(\AA^{3}\right)$ & 124.2 & 124.9 & 0.6 & Vol. $\left(\AA^{3}\right)$ & 638.0 & 639.2 & 0.2 \\
\hline
\end{tabular}


Table S2. Comparison of the PBC-GEBF-B97-2/pcSseg-2 and experimental data NMR chemical shifts (in ppm) for ${ }^{1} \mathbf{H}$-containing species.

\begin{tabular}{ccrrr} 
crystals & element & exp. & PBC-GEBF & \multicolumn{1}{c}{$\Delta$} \\
L-glutamic acid & COOH & 16.0 & 15.4 & -0.6 \\
3-deazaauracil & OH & 13.5 & 13.3 & -0.2 \\
& COOH & 12.3 & 14.2 & 1.9 \\
aspirin (form I) & Ph-H & 7.2 & 7.0 & -0.2 \\
& CH 3 & 1.4 & 0.6 & -0.8 \\
& $(\mathrm{~N}-\mathbf{H}) \mathrm{H} 1$ & 11.2 & 11.2 & 0.0 \\
uracil & $(\mathrm{N}-\mathbf{H}) \mathrm{H} 2$ & 10.8 & 11.5 & 0.7 \\
& $(\mathrm{C}-\mathbf{H}) \mathrm{H} 3$ & 7.5 & 7.5 & 0.0 \\
& $(\mathrm{C}-\mathbf{H}) \mathrm{H} 4$ & 6.0 & 5.8 & -0.2 \\
\hline
\end{tabular}


Table S3. Comparison of the PBC-GEBF-B97-2/pcSseg-2 and experimental data NMR chemical shifts (in ppm) for ${ }^{13}$ C-containing species.

\begin{tabular}{|c|c|c|c|c|}
\hline crystals & element & exp. & PBC-GEBF & $\Delta$ \\
\hline carbon dioxide & $\mathrm{O}=\mathrm{C}=\mathrm{O}$ & $133 \pm 3$ & 131.1 & -1.7 \\
\hline ethylene & $\mathrm{C}=\mathbf{C}$ & $126 \pm 3$ & 126.7 & 0.7 \\
\hline \multirow[t]{5}{*}{ acetylene } & $\mathrm{C} \equiv \mathbf{C}$ & $70 \pm 3$ & 70.1 & 0.1 \\
\hline & $\mathrm{C} 1$ & 122.1 & 120.9 & -1.2 \\
\hline & $\mathrm{C} 2$ & 152.9 & 155.8 & 2.9 \\
\hline & C3 & 125.5 & 126.2 & 0.7 \\
\hline & C4 & 138.3 & 143.9 & 5.6 \\
\hline \multirow[t]{9}{*}{ aspirin (form I) } & $\mathrm{C} 5$ & 127.6 & 131.3 & 3.7 \\
\hline & C6 & 134.8 & 138.0 & 3.2 \\
\hline & C7 & 170.7 & 176.9 & 6.2 \\
\hline & C8 & 171.9 & 181.5 & 9.6 \\
\hline & C9 & 19.8 & 17.8 & -2.0 \\
\hline & $(\mathbf{C}=\mathrm{O}) \mathrm{C} 1$ & 170.9 & 171.8 & 0.9 \\
\hline & $(\mathbf{C}=\mathrm{O}) \mathrm{C} 2$ & 151.7 & 152.3 & 0.6 \\
\hline & $(\mathbf{C}-\mathrm{H}) \mathrm{C} 3$ & 147.0 & 151.9 & 4.9 \\
\hline & $(\mathbf{C}-\mathrm{H}) \mathrm{C} 4$ & 99.9 & 98.4 & -1.5 \\
\hline L-glycine & $\mathbf{C}(\mathrm{O})$ & $176.2 \pm 0.2$ & 180.6 & 4.4 \\
\hline \multirow[t]{2}{*}{$(\alpha$-form) } & $\mathbf{C}_{\alpha}$ & $43.5 \pm 0.2$ & 39.5 & 4.0 \\
\hline & $\mathbf{C}(\mathrm{O})$ & $176 \pm 3$ & 182.8 & 6.8 \\
\hline \multirow[t]{4}{*}{ L-alanine } & $\mathbf{C}_{\alpha}$ & $50 \pm 2$ & 48.2 & -1.8 \\
\hline & $\mathbf{C}_{\beta}$ & $19 \pm 2$ & 14.7 & -4.3 \\
\hline & $\mathbf{C}(\mathrm{O})$ & $176 \pm 3$ & 180.6 & 4.6 \\
\hline & $\mathbf{C}_{\alpha}$ & $62 \pm 2$ & 56.9 & -5.1 \\
\hline \multirow[t]{4}{*}{ L-valine } & $\mathbf{C}_{\beta}$ & $32 \pm 2$ & 28.2 & -3.8 \\
\hline & $\mathbf{C}_{\gamma}$ & $22 \pm 2$ & 15.8 & -6.2 \\
\hline & $\mathbf{C}_{\delta}$ & $20 \pm 4$ & 13.5 & -6.5 \\
\hline & $\mathbf{C}(\mathrm{O})$ & $175 \pm 3$ & 180.2 & 5.2 \\
\hline \multirow[t]{2}{*}{ L-serine } & $\mathbf{C}_{\alpha}$ & $56 \pm 2$ & 57.8 & 1.8 \\
\hline & $\mathbf{C}_{\beta}$ & $63 \pm 2$ & 60.2 & -2.8 \\
\hline
\end{tabular}


Table S4. Comparison of the PBC-GEBF-B97-2/pcSseg-2 and experimental data NMR chemical shifts (in ppm) for ${ }^{15} \mathbf{N}$-containing species.

\begin{tabular}{|c|c|c|c|c|}
\hline crystals & element & exp. & PBC-GEBF & $\Delta$ \\
\hline borane & $\mathbf{N}-\mathrm{B}$ & -364 & -374.3 & -10.3 \\
\hline \multirow{2}{*}{ imidazole } & $\mathbf{N}$ & -132 & -135.4 & -3.4 \\
\hline & $\mathrm{HN}$ & -208 & -202.0 & 6.0 \\
\hline \multirow{2}{*}{ uracil } & $\mathbf{N} 1$ & -221 & -224.3 & -3.3 \\
\hline & $\mathbf{N} 3$ & -245 & -239.2 & 5.8 \\
\hline \multirow{2}{*}{ thymine } & $\mathbf{N} 1$ & -222 & -225.1 & -3.1 \\
\hline & $\mathbf{N} 3$ & -251 & -252.4 & -1.4 \\
\hline \multirow{5}{*}{ adenine } & N1 & -158 & -155.1 & 2.9 \\
\hline & $\mathbf{N} 3$ & -167 & -169.7 & -2.7 \\
\hline & $\mathbf{N} 7$ & -149 & -143.5 & 5.5 \\
\hline & N9 & -221 & -219.5 & 1.5 \\
\hline & $\mathbf{N H}_{2}$ & -291 & -290.1 & 0.9 \\
\hline \multirow{3}{*}{ cytosine } & $\mathbf{N} 1$ & -231 & -231.3 & -0.3 \\
\hline & $\mathbf{N} 3$ & -176 & -179.8 & -3.8 \\
\hline & $\mathbf{N H}_{2}$ & -287 & -291.4 & -4.4 \\
\hline \multirow{5}{*}{ guanine } & N1 & -231 & -236.1 & -5.1 \\
\hline & N3 & -206 & -207.1 & -1.1 \\
\hline & $\mathbf{N} 7$ & -146 & -147.3 & -1.3 \\
\hline & N9 & -229 & -229.1 & -0.1 \\
\hline & $\mathbf{N H}_{2}$ & -303 & -306.4 & -3.4 \\
\hline L-alanine & NH4 & -337.1 & -347.5 & -10.4 \\
\hline L-glycine $(\alpha)$ & NH4 & -346.3 & -356.2 & -10.9 \\
\hline
\end{tabular}


Table S5. Comparison of the PBC-GEBF-B97-2/pcSseg-2 and experimental data NMR chemical shifts (in ppm) for ${ }^{17} \mathbf{O}$-containing species.

\begin{tabular}{ccccc} 
crystals & O & exp. & PBC-GEBF & \multicolumn{1}{c}{$\Delta$} \\
urea & C=O & $179 \pm 1$ & 170.6 & -8.4 \\
L-alanine & O1 & 284 & 292.9 & 8.9 \\
& O2 & 260.5 & 264.5 & 4.0 \\
L-valine & $\mathbf{O} 1$ & $287 \pm 1$ & 286.9 & -0.1 \\
& $\mathbf{O} 2$ & $280 \pm 1$ & 285.3 & 5.3 \\
thymine & $\mathbf{O} 3$ & $313 \pm 1$ & 330.1 & 17.1 \\
cytosine & $\mathbf{O} 4$ & $264 \pm 1$ & 251.7 & -12.3 \\
guanine & $\mathbf{O} 1$ & $200 \pm 5$ & 195.2 & -4.8 \\
& $\mathbf{O} 2$ & $325 \pm 5$ & 328.6 & 3.6 \\
uracil & $\mathbf{O}$ & $230 \pm 5$ & 226.3 & -3.7 \\
& $\mathbf{O}$ & $230 \pm 5$ & 224.6 & -5.4 \\
& $\mathbf{O} 2$ & $275 \pm 5$ & 258.0 & -17.0 \\
& $\mathbf{O} 1$ & $215 \pm 1$ & 247.8 & 2.8 \\
aspirin (form I) & $\mathbf{O} 2$ & $273 \pm 1$ & 283.2 & -9.8 \\
& $\mathbf{O 3}$ & $203 \pm 1$ & 179.4 & -23.6 \\
& $\mathbf{O} 4$ & $369 \pm 1$ & 375.2 & 6.2 \\
\hline
\end{tabular}


Validation of the PBC-GEBF Method for Solution Systems.

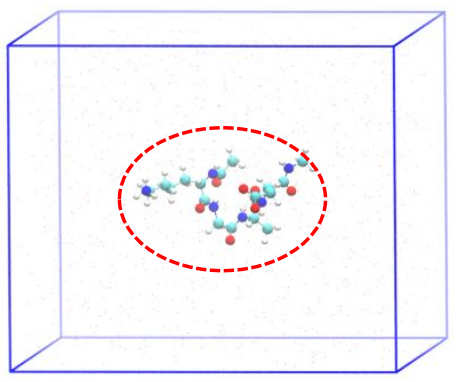

(a)

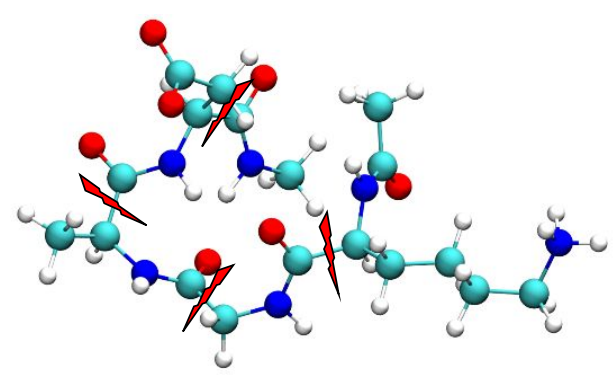

(b)

Figure S3. (a) An MD simulation box for a model tetrapeptide (ACE-Lys-Gly-Ala-Asp-NME) in water (in red dot for clarity). The Lys residue is positively charged (+1) and the Asp residue is negatively charged ( -1$)$. Classical MD simulation details are skipped here. (b) Four $\mathrm{C}-\mathrm{C}$ single bonds are cut (in red flash) for the model tetrapeptide. Color code: $\mathrm{H}$ in white, $\mathrm{C}$ in grey, $\mathrm{N}$ in blue, and $\mathrm{O}$ in red. 
Table S6. Comparison of the "molecule-based" (old) and the "fragment-based" (new) PBC-GEBF method in predicting NMR chemical shieldings (in ppm) for ACE-Lys-Gly-Ala-Asp-NME at the B97-2/pcSseg-2 level.

\begin{tabular}{|c|c|c|c|c|c|c|c|c|c|}
\hline NO. & element & old & new & dev. & NO. & element & old & new & dev. \\
\hline 1 & $\mathrm{H}$ & 28.98 & 28.98 & 0.00 & 33 & $\mathrm{H}$ & 27.70 & 27.72 & 0.02 \\
\hline 2 & $\mathrm{C}$ & 160.10 & 160.08 & -0.02 & 34 & $\mathrm{C}$ & 2.28 & 1.86 & -0.42 \\
\hline 3 & $\mathrm{H}$ & 29.39 & 29.40 & 0.01 & 35 & $\mathrm{O}$ & -71.62 & -71.24 & 0.38 \\
\hline 4 & $\mathrm{H}$ & 29.74 & 29.74 & 0.00 & 36 & $\mathrm{~N}$ & 117.24 & 117.59 & 0.34 \\
\hline 5 & $\mathrm{C}$ & 12.69 & 12.67 & -0.02 & 37 & $\mathrm{H}$ & 26.10 & 26.04 & -0.06 \\
\hline 6 & $\mathrm{O}$ & -4.22 & -4.54 & -0.32 & 38 & $\mathrm{C}$ & 121.29 & 121.17 & -0.12 \\
\hline 7 & $\mathrm{~N}$ & 109.58 & 109.90 & 0.32 & 39 & $\mathrm{H}$ & 27.43 & 27.38 & -0.05 \\
\hline 8 & $\mathrm{H}$ & 24.62 & 24.63 & 0.01 & 40 & $\mathrm{C}$ & 162.60 & 162.51 & -0.09 \\
\hline 9 & $\mathrm{C}$ & 128.46 & 128.56 & 0.10 & 41 & $\mathrm{H}$ & 29.32 & 29.27 & -0.05 \\
\hline 10 & $\mathrm{H}$ & 26.97 & 26.99 & 0.02 & 42 & $\mathrm{H}$ & 30.52 & 30.50 & -0.02 \\
\hline 11 & $\mathrm{C}$ & 139.94 & 139.91 & -0.03 & 43 & $\mathrm{H}$ & 29.63 & 29.64 & 0.01 \\
\hline 12 & $\mathrm{H}$ & 29.74 & 29.75 & 0.01 & 44 & $\mathrm{C}$ & -8.66 & -9.07 & -0.41 \\
\hline 13 & $\mathrm{H}$ & 29.49 & 29.52 & 0.03 & 45 & $\mathrm{O}$ & -50.03 & -49.02 & 1.01 \\
\hline 14 & $\mathrm{C}$ & 154.08 & 154.08 & 0.00 & 46 & $\mathrm{~N}$ & 110.64 & 110.88 & 0.24 \\
\hline 15 & $\mathrm{H}$ & 29.44 & 29.47 & 0.03 & 47 & $\mathrm{H}$ & 25.31 & 25.30 & -0.01 \\
\hline 16 & $\mathrm{H}$ & 29.57 & 29.59 & 0.02 & 48 & $\mathrm{C}$ & 125.33 & 125.40 & 0.07 \\
\hline 17 & $\mathrm{C}$ & 152.99 & 153.03 & 0.04 & 49 & $\mathrm{H}$ & 26.75 & 26.77 & 0.02 \\
\hline 18 & $\mathrm{H}$ & 29.89 & 29.90 & 0.01 & 50 & $\mathrm{C}$ & 147.26 & 147.32 & 0.06 \\
\hline 19 & $\mathrm{H}$ & 29.48 & 29.48 & 0.00 & 51 & $\mathrm{H}$ & 29.00 & 28.97 & -0.03 \\
\hline 20 & $\mathrm{C}$ & 142.56 & 142.53 & -0.03 & 52 & $\mathrm{H}$ & 28.38 & 28.35 & -0.03 \\
\hline 21 & $\mathrm{H}$ & 27.70 & 27.70 & 0.00 & 53 & $\mathrm{C}$ & 1.68 & 1.75 & 0.07 \\
\hline 22 & $\mathrm{H}$ & 28.22 & 28.27 & 0.05 & 54 & $\mathrm{O}$ & 2.91 & 1.49 & -1.42 \\
\hline 23 & $\mathrm{~N}$ & 208.08 & 207.98 & -0.10 & 55 & $\mathrm{O}$ & -17.68 & -17.38 & 0.30 \\
\hline 24 & $\mathrm{H}$ & 25.64 & 25.61 & -0.03 & 56 & $\mathrm{C}$ & 6.39 & 5.78 & -0.61 \\
\hline 25 & $\mathrm{H}$ & 27.06 & 27.11 & 0.05 & 57 & $\mathrm{O}$ & 7.33 & 9.59 & 2.26 \\
\hline 26 & $\mathrm{H}$ & 24.31 & 24.30 & -0.01 & 58 & $\mathrm{~N}$ & 133.61 & 133.61 & 0.00 \\
\hline 27 & $\mathrm{C}$ & -3.57 & -3.78 & -0.21 & 59 & $\mathrm{H}$ & 25.57 & 25.46 & -0.11 \\
\hline 28 & $\mathrm{O}$ & -50.95 & -51.03 & -0.08 & 60 & $\mathrm{C}$ & 152.99 & 153.05 & 0.06 \\
\hline 29 & $\mathrm{~N}$ & 132.15 & 132.71 & 0.56 & 61 & $\mathrm{H}$ & 27.65 & 27.63 & -0.02 \\
\hline 30 & $\mathrm{H}$ & 23.72 & 23.67 & -0.05 & 62 & $\mathrm{H}$ & 27.47 & 27.49 & 0.02 \\
\hline 31 & $\mathrm{C}$ & 137.21 & 137.08 & -0.13 & 63 & $\mathrm{H}$ & 28.75 & 28.76 & 0.01 \\
\hline 32 & $\mathrm{H}$ & 26.92 & 26.86 & -0.06 & & & & & \\
\hline $\mathbf{M S E}^{a}$ & & 0.03 & & & & & & & \\
\hline $\mathbf{M U E}^{b}$ & & 0.17 & & & & & & & \\
\hline
\end{tabular}

${ }^{a}$ MSE: mean signed error. ${ }^{b}$ MUE: mean unsigned error. 
Validation of PBC-GEBF NMR $J$-Couplings.

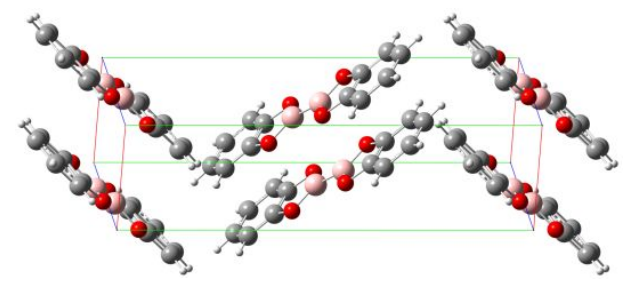

(1)

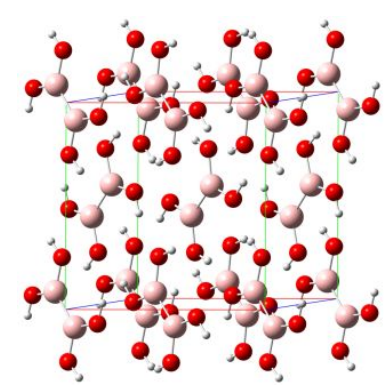

(3)

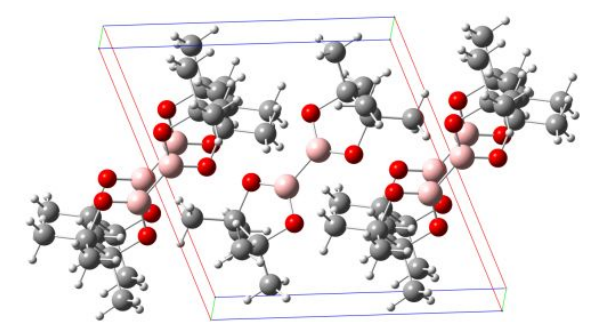

(2)

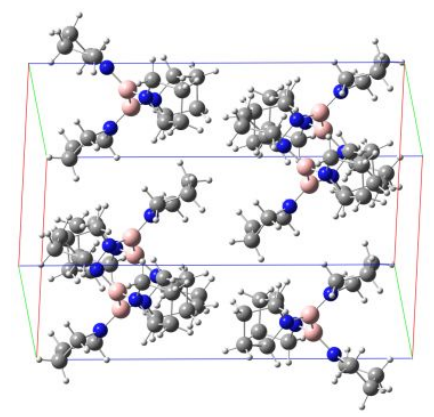

(4)

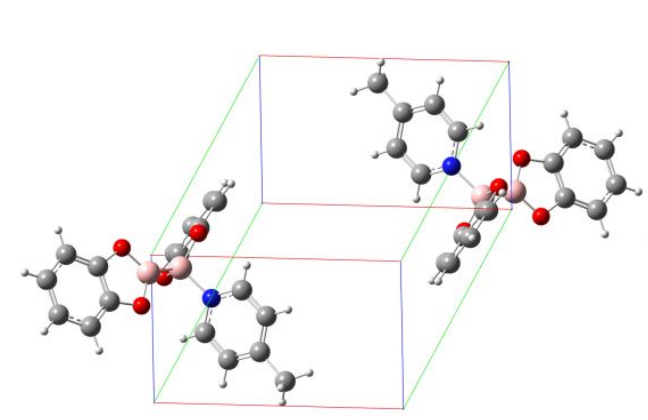

(5)

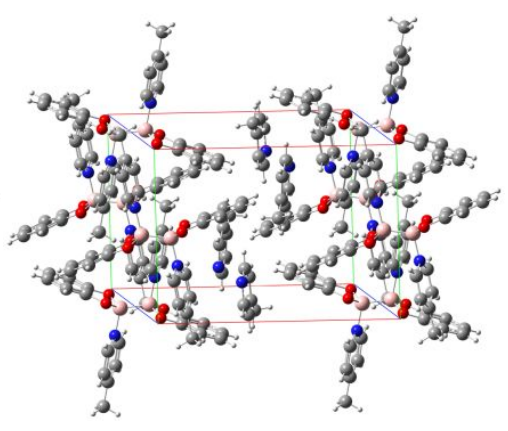

(6)

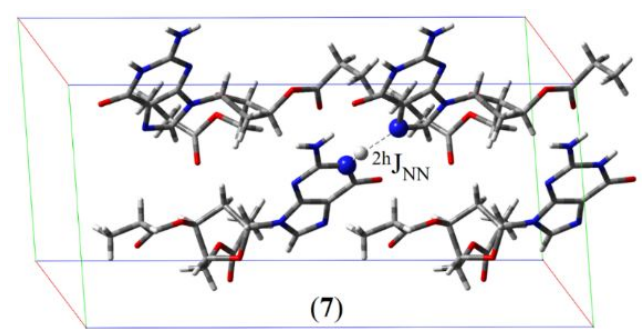

Figure S4. Six B-B-containing molecular crystals: ${ }^{1-5}$ (1) bis(catecholato) diboron, (2) bis(pinacolato) diboron, (3) tetrahydroxydiboron, (4) tetrakis(pyrrolidino) diborane, (5) [bis(catecholato)diboron] picoline, and (6) [bis(catecholato)diboron] dipicoline with small molecules. A $1 \times 1 \times 2$ supercell of the optimized structure of molecular crystal (7) ${ }^{6}$ with two inequivalent molecules in a primitive unit cell and a total of 96 atoms. Color code: $\mathrm{H}$ in white, $\mathrm{B}$ in pink, $\mathrm{C}$ in grey, $\mathrm{N}$ in blue, and $\mathrm{O}$ in red. 
Table S7. Comparison of $J$-couplings (in Hz) obtained from PBC-GEBF-X (X = B3LYP, B97-2, and PBE) computations $^{a}$ and the experiment.

\begin{tabular}{crrrr} 
NO. & B3LYP $^{b}$ & B97-2 $^{b}$ & PBE $^{b}$ & exp. $^{c, d}$ \\
1 & 152 & 150 & 144 & $136 \pm 1$ \\
2 & 130 & 127 & 121 & $120 \pm 2$ \\
3 & 115 & 124 & 119 & $121 \pm 3$ \\
4 & 92 & 90 & 97 & $98 \pm 2$ \\
5 & 129 & 127 & 119 & $115 \pm 4$ \\
6 & 108 & 104 & 96 & $108 \pm 1$ \\
7 & 9.0 & 8.7 & 8.5 & $7.4 \pm 0.4$ \\
& & & & \\
MSE $^{e}$ & 4.2 & 3.6 & -0.1 & \\
MUE $^{f}$ & 7.7 & 7.0 & 4.2 & \\
\hline
\end{tabular}

${ }^{a}$ Full lattice relaxations for 1-7 were carried out at the PBC-GEBF-PBE-D3(BJ)/6-31G(d,p) level. ${ }^{b} \mathrm{~A}$ generic basis set: 6-31G for $\mathrm{H}$ and $\mathrm{C}$; pcJ-2 for B, N, and O. ${ }^{c} \operatorname{Ref} 7 .{ }^{d} \operatorname{Ref} 8 .{ }^{e} \mathbf{M S E}$ : mean signed error. ${ }^{f} \mathbf{M U E}$ : mean unsigned error. 
Validation of PBC-GEBF NMR electric field gradient (EFG) tensors.

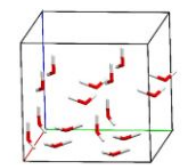

(a) Ih

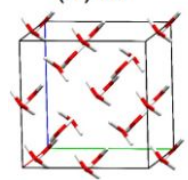

(f) VII

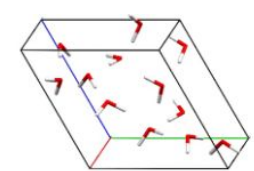

(b) II

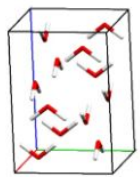

(g) VIII

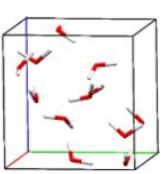

(c) III

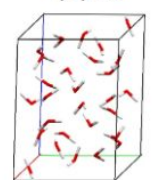

(h) XII

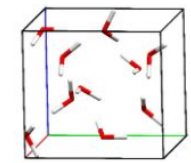

(d) IV

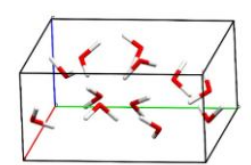

(i) XIV

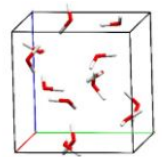

(e) IX

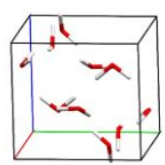

(j) $\mathrm{XV}$

Figure S5. Ten ice polymorphs (structures are taken from ref 9) used for the validation of PBC-GEBF.

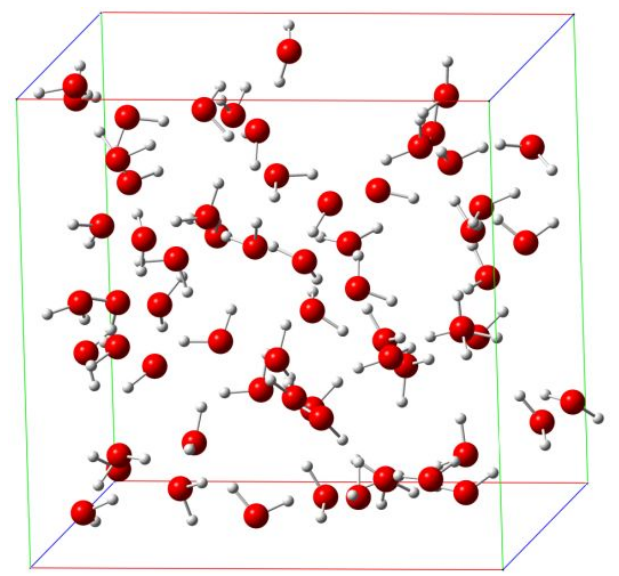

Figure S6. One of the ten water boxes $\left(\mathrm{H}_{2} \mathrm{O}\right)_{64}$ used for the validation of PBC-GEBF.

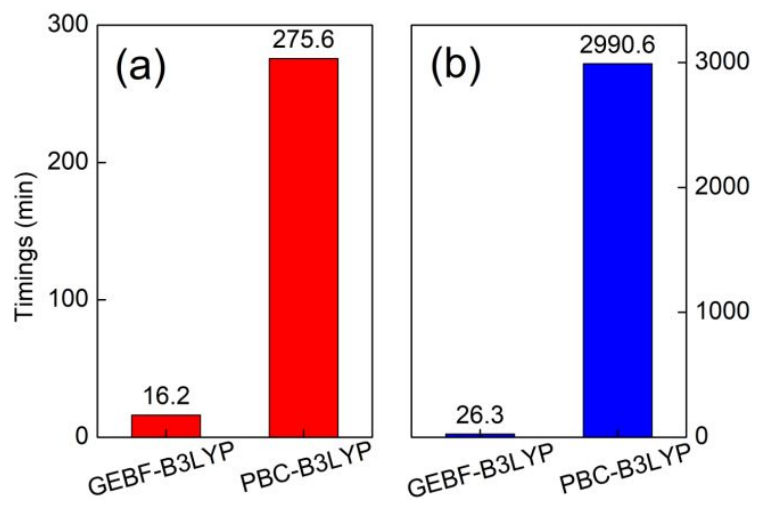


Figure S7. Comparison of average timings (in terms of wall time at a single computing node) for (a) ten ice polymorphs and (b) ten water boxes $\left(\mathrm{H}_{2} \mathrm{O}\right)_{64}$. The 6-31G(d,p) basis set was used.

Table S8. Root mean squared deviations (RMSDs) between PBC-GEBF-B3LYP/6-31G(d,p) and PBC-B3LYP/6$31 \mathrm{G}(\mathrm{d}, \mathrm{p})$ electric field gradient $(\mathrm{EFG})$ tensor components of ${ }^{17} \mathrm{O}$ and ${ }^{2} \mathrm{H}$ in ten ice polymorphs.

\begin{tabular}{ccccccc} 
& \multicolumn{3}{c}{${ }^{17} \mathrm{O}$ RMSD $(\mathrm{MHz})$} & & \multicolumn{3}{c}{${ }^{2} \mathrm{H}$ RSD $(\mathrm{KHz})$} \\
\cline { 2 - 6 } Ih & $x x$ & $y y$ & $z z$ & $x x$ & $y y$ & $z z$ \\
II & 0.13 & 0.75 & 0.19 & 0.85 & 0.34 & 1.16 \\
III & 0.09 & 0.06 & 0.12 & 0.85 & 0.80 & 1.09 \\
IV & 0.05 & 0.09 & 0.13 & 0.63 & 0.80 & 0.69 \\
IX & 0.06 & 0.08 & 0.07 & 0.73 & 0.83 & 1.08 \\
VII & 0.09 & 0.08 & 0.16 & 0.87 & 1.87 & 1.37 \\
VIII & 0.17 & 0.09 & 0.08 & 1.17 & 2.21 & 1.38 \\
XII & 0.15 & 0.08 & 0.07 & 1.36 & 2.37 & 1.25 \\
XIV & 0.15 & 0.07 & 0.16 & 1.89 & 1.44 & 1.06 \\
XV & 0.04 & 0.08 & 0.11 & 1.31 & 1.90 & 1.88 \\
\hline
\end{tabular}

Table S9. Root mean squared deviations (RMSDs) between PBC-GEBF-B3LYP/6-31G(d,p) and PBC-B3LYP/6$31 \mathrm{G}(\mathrm{d}, \mathrm{p})$ electric field gradient $(\mathrm{EFG})$ tensor components of ${ }^{17} \mathrm{O}$ and ${ }^{2} \mathrm{H}$ in ten water boxes $\left(\mathrm{H}_{2} \mathrm{O}\right)_{64}$.

\begin{tabular}{ccccccc} 
& \multicolumn{3}{c}{${ }^{17} \mathrm{O}$ RMSD $(\mathrm{MHz})$} & \multicolumn{3}{c}{${ }^{2} \mathrm{H}$ RMSD $(\mathrm{KHz})$} \\
\cline { 2 - 7 } 1 & $x x$ & $y y$ & $z z$ & $x x$ & $y y$ & $z z$ \\
2 & 0.08 & 0.04 & 0.06 & 6.25 & 3.39 & 3.62 \\
3 & 0.06 & 0.03 & 0.06 & 4.78 & 3.08 & 2.64 \\
4 & 0.07 & 0.03 & 0.06 & 6.76 & 3.70 & 3.71 \\
5 & 0.06 & 0.03 & 0.06 & 6.74 & 3.78 & 3.65 \\
6 & 0.06 & 0.03 & 0.05 & 6.56 & 3.33 & 4.02 \\
7 & 0.06 & 0.04 & 0.05 & 6.83 & 3.67 & 4.02 \\
8 & 0.05 & 0.03 & 0.05 & 7.22 & 3.69 & 4.07 \\
9 & 0.06 & 0.03 & 0.05 & 6.49 & 3.49 & 3.62 \\
10 & 0.05 & 0.03 & 0.05 & 6.35 & 3.57 & 3.66 \\
\hline
\end{tabular}


Table S10. Comparison of predicted and experimental NMR parameters of gas-phase water.

\begin{tabular}{lcc} 
NMR parameters & B3LYP/pcJ-2 & exp. $^{10-13}$ \\
$\sigma_{\mathrm{O}}(\mathrm{g})(\mathrm{ppm})$ & 326.6 & 328.3 \\
$\sigma_{\mathrm{H}}(\mathrm{g})(\mathrm{ppm})$ & 30.9 & 30.0 \\
${ }^{1} J\left({ }^{17} \mathrm{O},{ }^{1} \mathrm{H}\right)(\mathrm{Hz})$ & -75.7 & -78.2 \\
$\chi_{\mathrm{O}}(\mathrm{MHz})$ & 10.7 & 10.2 \\
$\chi_{\mathrm{H}}(\mathrm{KHz})$ & 310.2 & 307.9 \\
$\eta_{\mathrm{O}}$ & 0.75 & 0.75 \\
$\eta_{\mathrm{H}}$ & 0.130 & 0.135 \\
\hline
\end{tabular}

Table S11. Comparison of isotropic shielding constants $\sigma(1)$, spin-spin ${ }^{1} J\left({ }^{17} \mathrm{O},{ }^{1} \mathrm{H}\right)$ and quadrupolar coupling $\chi$ constants. ${ }^{a}$

\begin{tabular}{cccccc} 
Frame & $\sigma_{\mathrm{O}}(\mathrm{l})(\mathrm{ppm})$ & $\sigma_{\mathrm{H}}(\mathrm{l})(\mathrm{ppm})$ & ${ }^{1} J\left({ }^{17} \mathrm{O},{ }^{1} \mathrm{H}\right)(\mathrm{Hz})$ & $\chi_{\mathrm{O}}(\mathrm{MHz})$ & $\chi_{\mathrm{H}}(\mathrm{KHz})$ \\
1 & 282.2 & 26.7 & -81.6 & 8.79 & 244.9 \\
2 & 282.2 & 26.8 & -80.8 & 8.83 & 244.0 \\
3 & 281.5 & 26.6 & -81.5 & 8.76 & 240.8 \\
& & & & & \\
avg. & 282.0 & 26.7 & -81.3 & 8.79 & 243.2 \\
\hline
\end{tabular}

${ }^{a}$ B3LYP/pcJ-2//(3) NNP-based (RPBE-D3) $)^{14}$ ab initio MD frames of $\left(\mathrm{H}_{2} \mathrm{O}\right)_{512}$. 


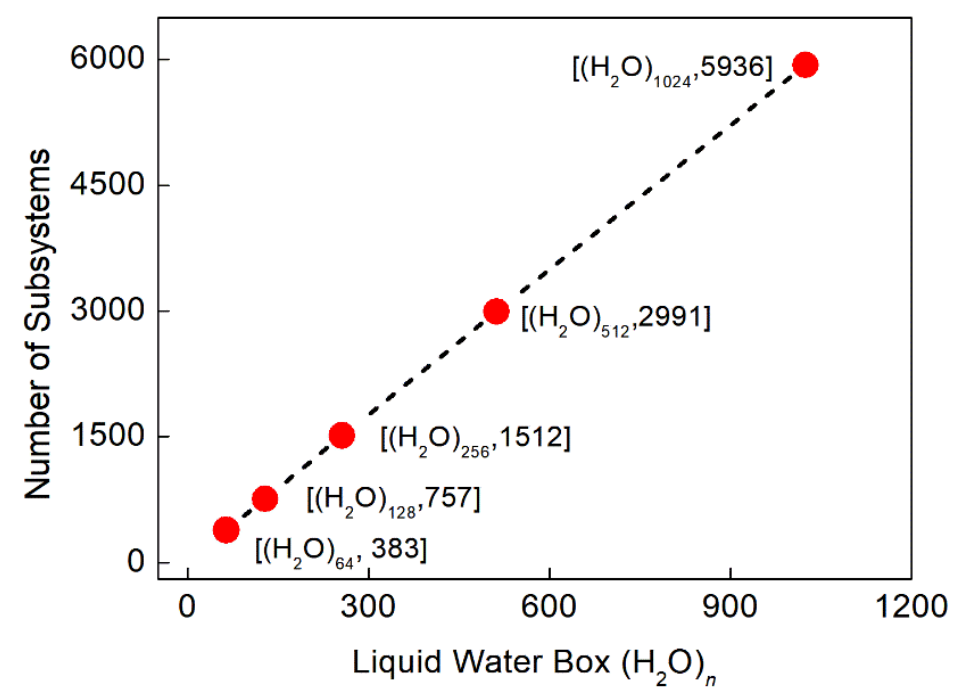

Figure S8. The total number of subsystems with respect to the number of water molecules in a unit cell. 


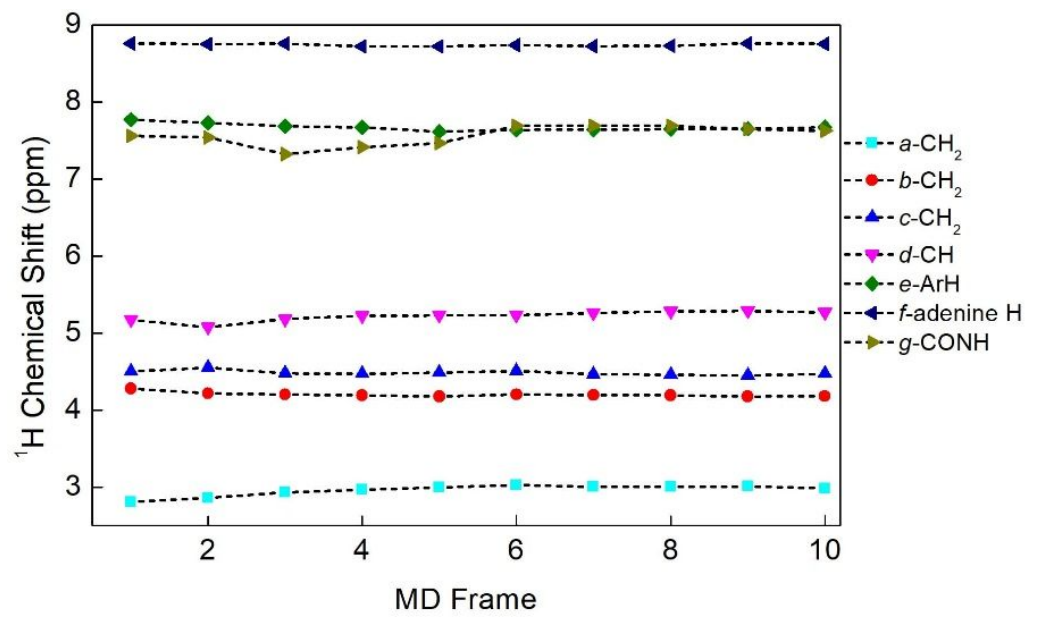

Figure S9. The plot of calculated chemical shifts for each ${ }^{1} \mathrm{H}$ nucleus with respect to the number of MD frames. All calculations were carried out at the PBC-GEBF-B97-2/pcSseg-2 level with the new "fragment-based" fragmentation strategy. 


\section{References}

(1) Clegg, W.; Elsegood, M. R. J.; Lawlor, F. J.; Norman, N. C.; Pickett, N. L.; Robins, E. G.; Scott, A. J.; Nguyen, P.; Taylor, N. J.; Marder, T. B. Structural Studies of Bis-Catecholate, Bis-Dithiocatecholate, and Tetraalkoxy Diborane(4) Compounds. Inorg. Chem. 1998, 37, 5289-5293.

(2) Kleeberg, C.; Crawford, A. G.; Batsanov, A. S.; Hodgkinson, P.; Apperley, D. C.; Cheung, M. S.; Lin, Z.; Marder, T. B. Spectroscopic and Structural Characterization of the CyNHC Adduct of $\mathrm{B}_{2} \mathrm{pin}_{2}$ in Solution and in the Solid State. $J$. Org. Chem. 2012, 77, 785-789.

(3) Baber, R. A.; Norman, N. C.; Orpen, A. G.; Rossi, J. The solid-state structure of diboronic acid, $\mathrm{B}_{2}(\mathrm{OH})_{4}$. New J. Chem. 2003, 27, 773-775.

(4) Ali, H. A.; Goldberg, I.; Srebnik, M. Tetra(pyrrolidino)diborane(4), $\left[\left(\mathrm{C}_{4} \mathrm{H}_{8} \mathrm{~N}\right)_{2} \mathrm{~B}\right]_{2}$, as a New Improved Alternative Synthetic Route to Bis(pinacolato)diborane(4) - Crystal Structures of the Intermediates. Eur. J. Inorg. Chem. 2002, $73-$ 78.

(5) Nguyen, P.; Dai, C.; Taylor, N. J.; Power, W. P.; Marder, T. B.; Pickett, N. L.; Norman, N. C. Lewis Base Adducts of Diboron Compounds: Molecular Structures of $\left[\mathrm{B}_{2}\left(\right.\right.$ cat $_{2}(4-$ picoline $\left.)\right]$ and $\left[\mathrm{B}_{2}(\text { cat })_{2}(4-\text { picoline })_{2}\right]\left(\right.$ cat $\left.=1,2-\mathrm{O}_{2} \mathrm{C}_{6} \mathrm{H}_{4}\right)$. Inorg. Chem. 1995, 34, 4290-4291.

(6) Giorgi, T.; Grepioni, F.; Manet, I.; Mariani, P.; Masiero, S.; Mezzina, E.; Pieraccini, S.; Saturni, L.; Spada, G. P.; Gottarelli, G. Gel-Like Lyomesophases Formed in Organic Solvents by Self-Assembled Guanine Ribbons. Chem.-Eur. J. 2002, 8, 2143-2152.

(7) Perras, F. A.; Bryce, D. L. Boron-boron $J$ coupling constants are unique probes of electronic structure: a solid-state NMR and molecular orbital study. Chem. Sci. 2014, 5, 2428-2437.

(8) Pham, T. N.; Griffin, J. M.; Masiero, S.; Lena, S.; Gottarelli, G.; Hodgkinson, P.; Filip, C.; Brown, S. P. Quantifying hydrogen-bonding strength: the measurement of ${ }^{2 \mathrm{~h}} \mathrm{~J}_{\mathrm{NN}}$ couplings in self-assembled guanosines by solid-state ${ }^{15} \mathrm{~N}$ spin-echo MAS NMR. Phys. Chem. Chem. Phys. 2007, 9, 3416-3423.

(9) Brandenburg, J. G.; Maas, T.; Grimme, S. Benchmarking DFT and semiempirical methods on structures and lattice energies for ten ice polymorphs. J. Chem. Phys. 2015, 142, 124104.

(10) Raynes, W. T. in Nuclear Magnetic Resonance, A Specialist Periodical Report; Royal Society of Chemistry, London, 1978.

(11) Komorovsky, S.; Repisky, M.; Malkin, E.; Ruud, K.; Gauss, J. Communication: The absolute shielding scales of oxygen and sulfur revisited. J. Chem. Phys. 2015, 142, 091102.

(12) Makulski, W. In Gas Phase NMR; Jackowski, K., Jaszuński, M., Eds.; The Royal Society of Chemistry: London, 2016; Chapter 5, pp 152-185.

(13) Verhoeven, J.; Dymanus, A.; Bluyssen, H. Hyperfine Structure of $\mathrm{HD}^{17} \mathrm{O}$ by Beam-Maser Spectroscopy. J. Chem. Phys. 1969, 50, 3330-3338.

(14) Morawietz, T.; Singraber, A.; Dellago, C.; Behler, J. How van der Waals interactions determine the unique properties of water. Proc. Natl. Acad. Sci. U.S.A. 2016, 113, 8368-8373. 